\title{
Development of composition-insensitive SMA thin films with high recovery strength and large recovery strain
}

\author{
S. Kajiwara
}

National Research Institute for Metals, 1-2-1 Sengen, Tsukuba 0047, Japan

\begin{abstract}
After reviewing the microstructures formed in Ti-rich Ti-Ni based thin films and the associated shape memory properties, it is shown that thin films of composition-insensitive shape memory alloys (SMA) can be developed by utilizing the formation of coherent bet thin plate precipitates. Ti-rich sputter-deposited thin films of Ti$\mathrm{Ni}-\mathrm{Cu}$ alloys are presented as such an example, which exhibit excellent shape memory properties of $500 \mathrm{MPa}$ recovery stress with about $5 \%$ recovery strain for the Ti-composition range of 51-54 at. \%. Necessary conditions for producing such thin films are (1) Ti-rich content, i.e., over 50 at. $\%$, (2) the amorphous initial state, (3) heat treatment at low temperatures near the crystallization temperature and (4) ternary alloy system. It is suggested that the same approach will be possible for the other ternary alloys such as $\mathrm{Ti}-\mathrm{Ni}-\mathrm{Pd}$, Ti-Ni-Hf, etc..
\end{abstract}

\section{INTRODUCTION}

Since the first successful attempt by Miyazaki et al. [1] in 1992 to produce Ti-Ni shape memory alloy (SMA) thin films by sputtering method, intensive studies on sputter-deposited Ti-Ni based thin films have been performed and it was reported that these thin films have almost the same shape memory properties as those of the bulk materials when the thin films were subjected to the similar heat treatments [2]. As is widely admitted, the final goal of those investigations is to apply SMA thin films for the micro-actuator of micro-machines. However, since the transformation temperatures of $\mathrm{Ti}-\mathrm{Ni}$ based alloys, which are major factors controlling shape memory properties, are extremely sensitive to alloy composition, it would be very difficult to produce a desired alloy composition of thin films by sputtering technique. Then a serious problem will arise on how to make the thin film that is fulfilled with the required shape memory properties. The present paper will demonstrate how this problem can be overcome. The only solution for this problem will be to utilize Ti-rich thin films which contain coherent thin plate precipitates of bodycentered tetragonal lattice (bct). The basic idea is that the excess $\mathrm{Ti}$ atoms are absorbed into those precipitates so that the matrix composition can be kept constant nearly at the stoichiometric composition (50 at. \% Ti), and, unlike $\mathrm{Ti}_{2} \mathrm{Ni}$ precipitates, those coherent bct precipitates are quite beneficial for obtaining good shape memory effect.

In 1996, Kajiwara et al.[3] reported that, if Ti-rich Ti-Ni amorphous films are directly heated at relatively low temperatures near the crystallization temperature, very thin coherent bct plate precipitates, which is a non-equilibrium phase, are formed on $\{100\}$ planes in the matrix and, owing to these precipitates, high recovery strength and large recovery strain in shape memory effect are obtained [3-6]. This is impossible with the bulk sample because the amorphous state cannot be realized even by extremely high speed splat cooling. Very recently it was found that, in the case of Ti-rich Ti-Ni-Cu alloys, much higher recovery strength is attained with very small transformation hysteresis by such heat treatment [7]. In the present paper, the author first describes in detail the microstructures of the bct precipitates revealed by conventional and high-resolution electron microscopy and, then, shows some of the essential points of the related shape memory properties. Finally the basic principle is drawn for designing composition insensitive SMA thin films with high recovery strength and large recovery strain. 


\section{MICROSTRUCTURES FORMED BY LOW-TEMPERATURE HEAT TREATMENTS}

The Ti-rich Ti-Ni based thin films that we have studied so far are as follows; for Ti-Ni binary alloy system, Ti-48.2Ni, Ti-47.3Ni and $\mathrm{Ti}-45.4 \mathrm{Ni}$ (at.\%), and for Ti-Ni-Cu ternary alloy system, Ti43.0Ni-6.2 Cu and Ti-40.0Ni-5.7Cu (at.\%). The thin films were prepared by sputtering on a substrate of glass or copper, using a radio frequency magnetron sputtering method. The film thickness was about 7

$\mu \mathrm{m}$. The as-sputtered films were all in the amorphous state and the heat treatments in the present work

were performed by heating directly from the amorphous state and kept at a given temperature for a certain time. The crystallization temperatures, Tc, for these alloys are all around $750 \mathrm{~K}$. The structure of the coherent precipitate mentioned in section 1 is schematically shown in Fig. 1. As depicted in this figure, the habit plane of the bct precipitate is $\{100\}$ and its c-axis is normal to the habit plane. The perfect lattice coherency between the matrix and the precipitate is maintained at the $\{100\}$ habit plane. The precipitate has a disc shape with 0.5-1.5 nm thickness and 5-10 nm diameter. The precipitate is Ti-rich and its bet lattice has very high tetragonalities of $\mathrm{c} / \mathrm{a}=1.1-1.4$. For formation of this bct precipitate, the following three conditions must be fulfilled. (1) The Ti content must be greater than 50 at.\%. (2) The initial state of the specimen must be amorphous. (3) The heating temperatures must be relatively low, near the crystallization temperature (Tc), and the heating to those temperatures must be done directly from the amorphous state. The distribution of these thin plate bct precipitates is quite different depending on a slightly different heating temperature as described in the following $[3,4,8,9]$.

\subsection{Heat treatment near Tc}

Fig. 2 shows an electron micrograph of thin plate precipitates taken with a conventional electron microscope, which were formed by heating at $745 \mathrm{~K}$ for $3.6 \mathrm{ks}$ in $\mathrm{Ti}-48.2 \mathrm{Ni}(\mathrm{Tc}=737 \mathrm{~K})$. The incident beam is parallel to $\langle 100\rangle$ of the $\mathrm{B} 2$ matrix. So the bct plate precipitates are seen in the edge-on orientation for those on $\{100\}$ habit planes parallel to the incident beam. The plate precipitates on the habit plane normal to the incident beam are not seen in this micrograph. For all the electron micrographs shown in this paper, the foil orientation is always the same to this case, i.e., the incident beam is parallel to $<100>$ of the $\mathrm{B} 2$ matrix. The bct plate precipitates are observable only with this orientation because the precipitate thickness is extremely small. Fig. 3 is a low magnification micrograph of high-resolution microscope images of the precipitates. The corresponding diffraction pattern is inserted in the figure. Weak spots between 110 spots are originated from the R-phase into which the B2 matrix has been transformed at $328 \mathrm{~K}$ during cooling to room temperature. Streaks along $<100>$ directions are due to the thin plate precipitates. In this figure we see strong dark contrast around the precipitate, which is attributed to the coherency strain between the precipitate and the matrix. Fig. 4(a) shows high-resolution microscope images revealing the atomic arrangement of the precipitates. Two plate precipitates are seen along (010) and (100) as indicated by arrows. We can clearly see the bend of the lattice images of $\{110\}$ planes at those precipitates. This kind of the bend is exactly the same as schematically illustrated in Fig. 1.

The measured bend angle is $9^{\circ}$ for the precipitate along the $(010)$ plane and, then, $c / a=1.38$ by equation

$c / d=\tan \theta\left(\theta=54^{\circ}\right)$. A further enlarged micrograph of this part is shown in Fig. 4 (b), which is an inverse

Fourier transform image of the lower part of Fig. 4(a). From the structure image in this figure, the tetragonality of this bct precipitate is directly measured to be 1.35 , which is in good agreement with the above-mentioned one evaluated by the bend angle of $\{110\}$ lattice planes. It should be noted that $\{110\}$ lattice planes neighbor to the precipitate are severely curved to keep the coherency with the precipitate. This is the origin for a large coherency strain associated with the precipitate. The thickness of the bct precipitate formed in this temperature regime is usually very small, about $0.3 \mathrm{~nm}$, i.e., one unit cell of the bct lattice as seen in Fig. 4(b). The same precipitates are formed for Ti-43.0Ni-6.2Cu (Tc=748 K) by heat treatment near $\mathrm{Tc}$, but, for $\mathrm{Ti}-47.3 \mathrm{Ni}(\mathrm{Tc}=752 \mathrm{~K})$ and $\mathrm{Ti}-40.0 \mathrm{Ni}-5.7 \mathrm{Cu}(\mathrm{Tc}=754 \mathrm{~K}), \mathrm{Ti}_{2} \mathrm{Ni}$ precipitates are also formed mixed with the bct precipitates. 


\subsection{Heat treatment below Tc}

Unlike a uniform distribution of bet precipitates in the case of the heat treatment near $T c$, bct precipitates are produced in clustered form in the case of the heat treatment below $\mathrm{Tc}$ (typically lower by $50 \mathrm{~K}$ than $\mathrm{Tc}$ ). These precipitates eventually constitute the boundaries of nanocrystals of which the orientations are exactly the same throughout a grain. Fig. 5 shows a conventional electron micrograph of the substructures in a grain formed by heating at $687 \mathrm{~K}(=\mathrm{Tc}-50 \mathrm{~K})$ for $10.8 \mathrm{ks}$ in Ti-48.2Ni. Mesh-like structures are seen in the grain and the grain boundary has a zigzag shape in line with those mesh-like structures. Although some areas have dark contrast while the others do not, it was proved by electron diffraction that the whole grain has the same orientation. A much higher magnification micrograph of a part of this grain is shown in Fig. 6, where a net-like pattern formed by bright or dark lines is seen. It is noted in this figure that these lines are aligned approximately parallel to either (100) or (010) planes and the size of each unit area surrounded by these lines is $20-40 \mathrm{~nm}$.

Fig. 7(a) shows a low magnification micrograph of high-resolution electron microscope images of the net-like structures such as seen in Fig. 6 and the region contacting with the amorphous that has not yet been crystallized (in the right corner of the micrograph). The dark or bright line contrasts along on (100) or $(010)$ are thin bet plate precipitates formed on these planes, which are viewed in parallel to their habit planes. The micrograph in Fig. 7(b) is an enlarged one of the upper right area of Fig. 7(a). A bct precipitate can be identified to exist at the place indicated an arrow in Fig. $7(\mathrm{~b})$ where $\{110\}$ lattice images are bent, forming a kind of the boundary between crystals $A$ and $B$. It is clearly seen in this micrograph that the $\{110\}$ lattice images are perfectly parallel for crystals $\mathrm{A}, \mathrm{B}$ and $\mathrm{C}$, which means that their orientations are exactly the same. This is true also for the other crystals in (a). It should be also noted that the perfect lattice coherency between the matrix and the bct precipitate is maintained as seen at the boundary between crystals A and B. Another important finding in Fig. 7(b) is that bet plate precipitates are also observed at the interface between the crystallized region and the amorphous region as indicated by arrows $P$ and $Q$ in Fig. 7(b). The lattice coherency between the precipitate and the crystallized region is well maintained also for this case. The formation of bct precipitate at the amorphous-crystal interface is a very important experimental fact for considering the formation mechanism of the nanocrystals below Tc. The size of the crystals bounded by the thin precipitates in Fig. 7 (a) is $20-40 \mathrm{~nm}$, so it is reasonably assumed that the regions delineated by line contrasts in Fig. 6 correspond to these nano-size crystals (i.e., nanocrystals) and small mesh-like regions in Fig. 5 also corresponds to such nanocrystals. One of the reason for this assumption is that their sizes are all the same, i.e., in the range of $20-40 \mathrm{~nm}$. In the micrograph of Fig. 7(a), bct plate precipitates are often seen to arrange shifted aside. This is considered as an effort for those plate precipitates to contact with the basically spherical nanocrystal as much as possible. That is, each nanocrystal with the approximately spherical shape is truncated by bct thin plate precipitates on $\{100\}$. The basically same nanocrystals were formed for Ti-43.0Ni-6.2Cu and Ti-40.0Ni$5.7 \mathrm{Cu}$ by heat treatments below $\mathrm{Tc}$ (lower by $30-50 \mathrm{~K}$ ), while, for Ti-47.3 Ni, randomly orientated $\mathrm{Ti}_{2} \mathrm{Ni}$ precipitates were also formed mixed with the nanocrystals bounded by bet precipitates. For Ti-45.4Ni $(\mathrm{Tc}=760 \mathrm{~K})$, the bet precipitate itself was not generated probably because $\mathrm{Ti}_{2} \mathrm{Ni}$ particles had been formed directly from the amorphous before the crystallization into B2 structure started, and then, the supersaturated state of $\mathrm{Ti}$ atoms had been already eliminated in the remaining amorphous region.

\subsection{Formation mechanism of bet precipitate}

When Ti-rich Ti-Ni amorphous samples are heated at Tc or at slightly higher temperatures, it is supposed that the as-crystallized region may be able to contain excess Ti atoms randomly for some time because of the rapid crystallization. However, after time has elapsed, these excess $\mathrm{Ti}$ atoms must be expelled from the B2 matrix that has a tight one to one Ti-Ni bonding. Those atoms are segregated on $\{100\}$ planes to form bet precipitates such as shown in Fig. 1. The tetragonal lattice is created due to strong repulsion 


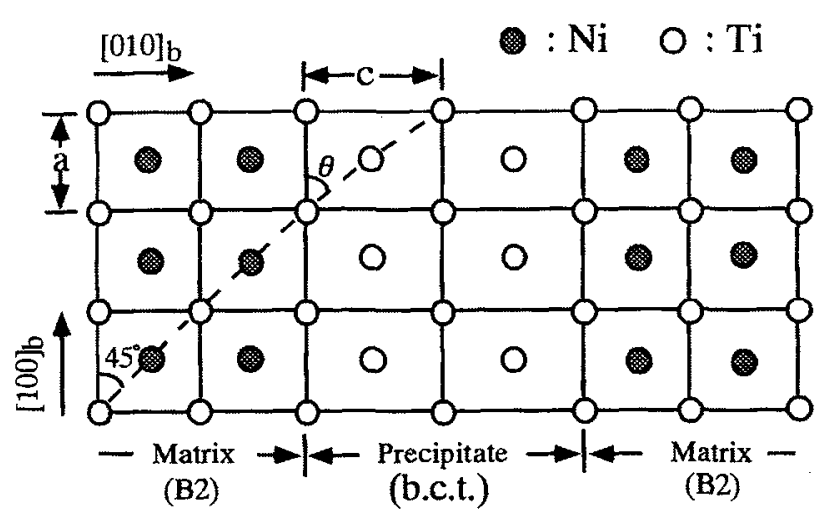

Figure 1: Schematic picture of Ti-rich bct precipitate with $(010)$ habit plane. The c-axis in bct lattice is normal to $(010)$ habit plane. The perfect lattice coherency is maintained on $(010)$ between the precipitate and the B2 matrix.

Figure 2: Conventional electron microscope micrograph of uniformly distributed bct plate precipitates in $\mathrm{Ti}-48.2 \mathrm{Ni}$. Heat treated at $745 \mathrm{~K}$ (near Tc) for $3.6 \mathrm{ks}$. The precipitates formed on $\{100\}$ are seen in the edge-on orientation.
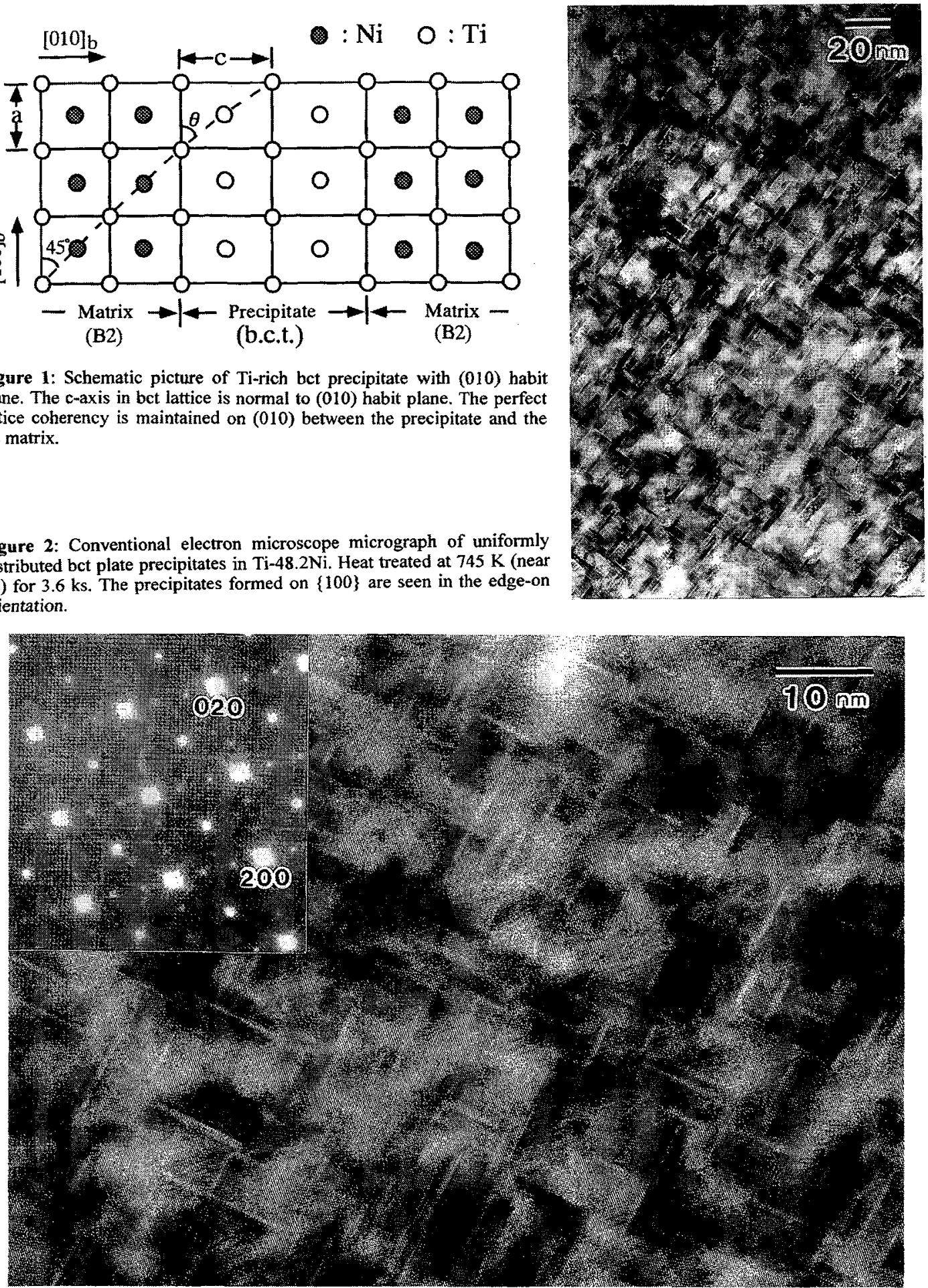

Figure 3: Low magnification micrograph of high-resolution electron microscope images of bct plate precipitates. The corresponding diffraction pattern with [001] zone axis is inserted. Note dark strain contrast around the precipitates. 
(a)

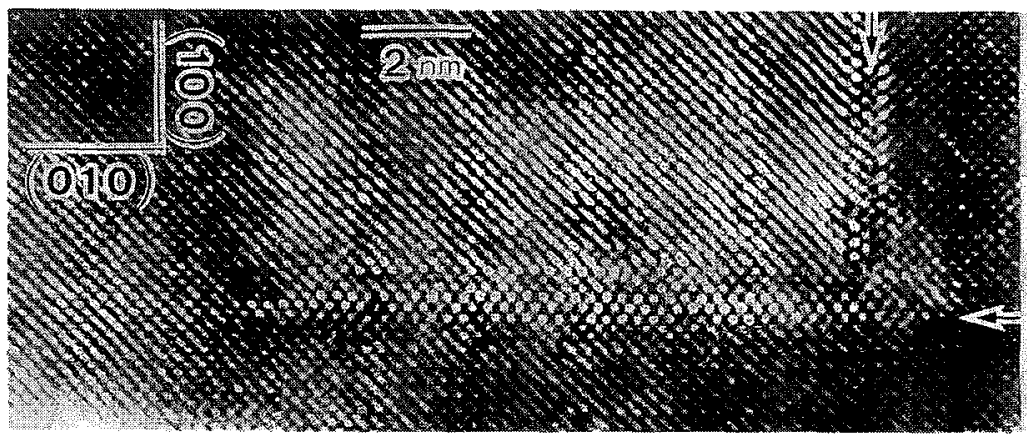

(b)

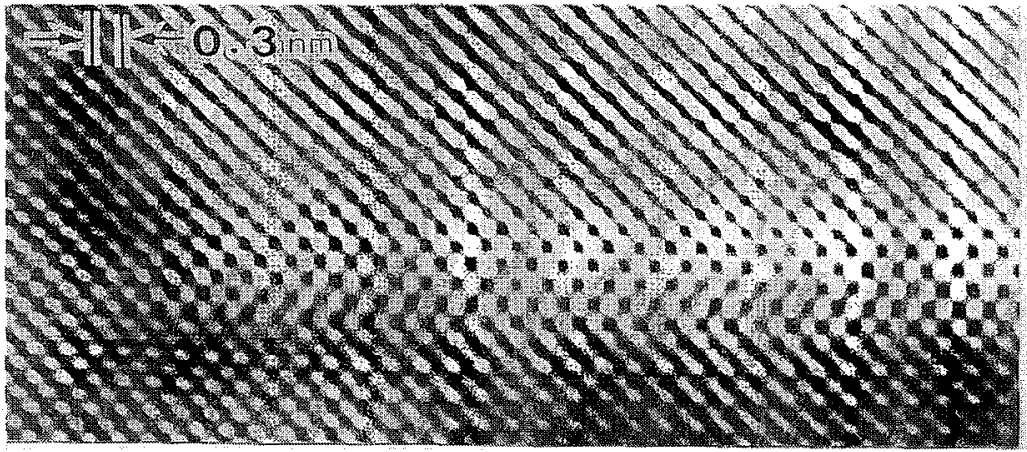

Figure 4: High resolution electron microscope images, showing atomic arrangements in the bct precipitates. Atom rows along [010] in the bct lattice of the precipitates are seen. (a) Two bct precipitates formed along (010) and (100) in the B2 matrix are indicated by arrows. (b) Enlarged micrograph of an inverse Fourier transform image of the lower part of (a). Atomic arrangements at the precipitate and its neighboring matrix are clearly visible.

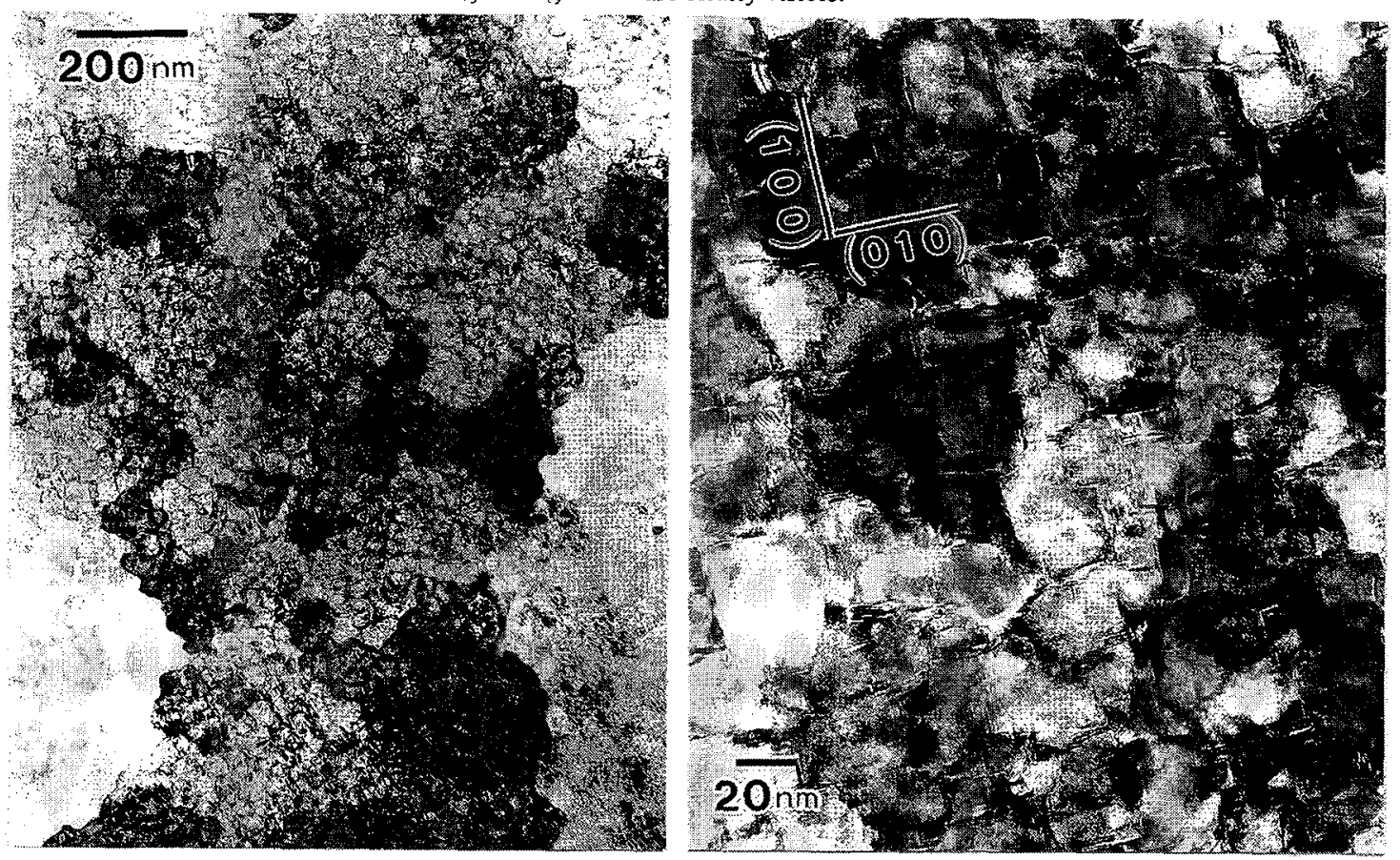

Figure 5: Conventional electron micrograph of nanocrystals bounded by Figure 6: Enlarged micrograph of a part of Fig. 5. bct precipitates in Ti- $48.2 \mathrm{Ni}$. Heat treated at $687 \mathrm{~K}(=\mathrm{Tc}-50 \mathrm{~K})$ for $10.8 \mathrm{ks}$. 

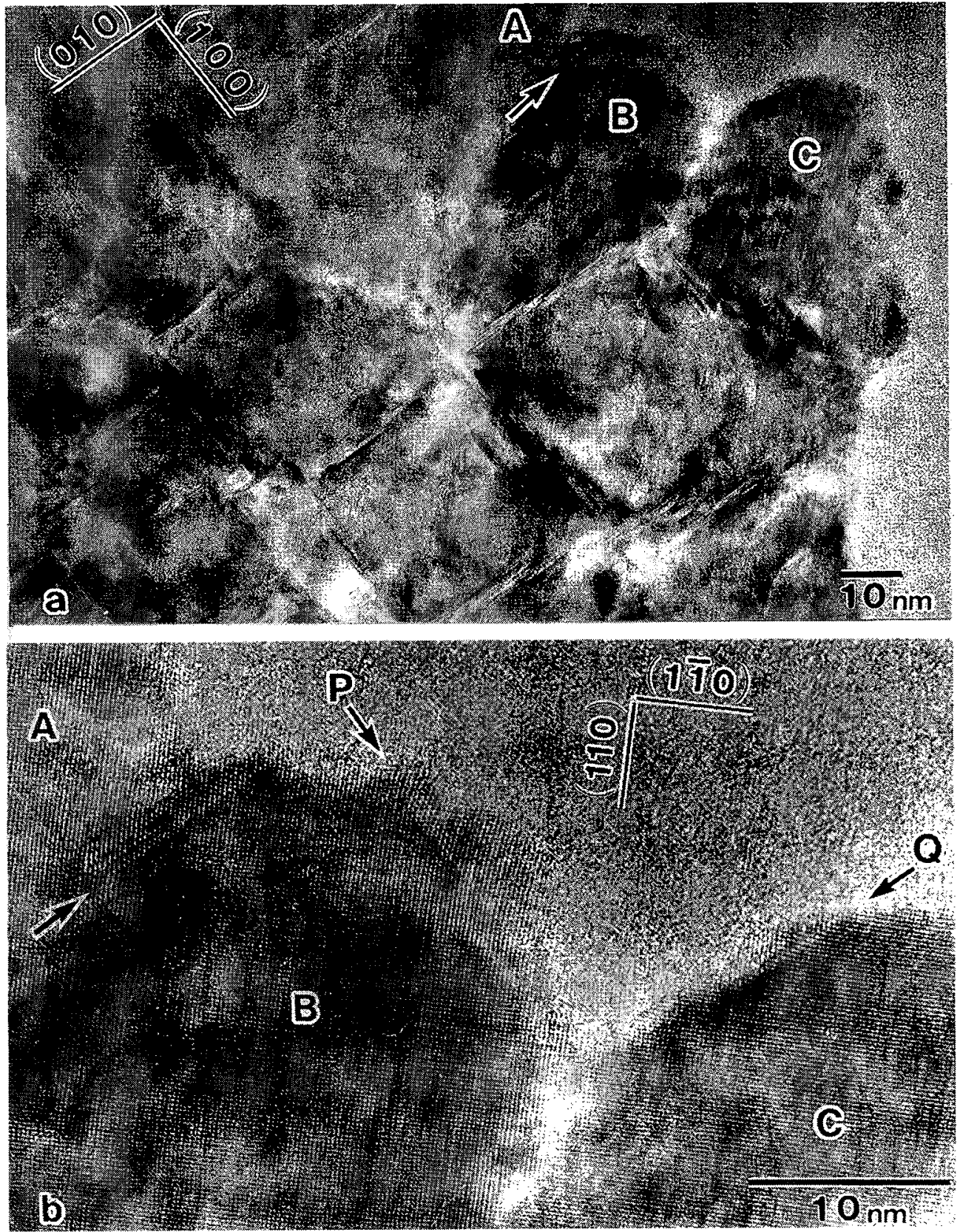

Figure 7: (a) Low magnification micrograph of high-resolution electron microscope images, showing nanocrystals of which the boundaries are made up of bct precipitates. (b) Enlarged micrograph of the upper right corner of (a). Plate precipitates of bct lattice are seen at the places indicated by arrows. Upper right region is amorphous. 
force between $\mathrm{Ti}$ atoms and a larger atom size of $\mathrm{Ti}$ (larger than $\mathrm{Ni}$ by $19 \%$ ). At these heating temperatures, diffusion of $\mathrm{Ti}$ atoms cannot occur enough to produce stable $\mathrm{Ti}_{2} \mathrm{Ni}$ precipitates.

At heating temperatures lower than Tc, the bcc phase generated from the Ti-rich amorphous will take an ordered B2 structure with the exact equiatomic composition at the time of crystallization because the Ti-Ni bond is very strong due to low temperature. An excess amount of $\mathrm{Ti}$ atoms will be then pushed away into the amorphous region near the advancing crystal-amorphous interface. As the interface advances, more and more $\mathrm{Ti}$ atoms will be accumulated in that amorphous region and, when the excess $\mathrm{Ti}$ content has reached a certain level, a Ti-rich bct precipitate is produced to eliminate the over-saturated $\mathrm{Ti}$ atoms in the amorphous. After this precipitate formation, a new B2 crystal will be generated at the front surface of the precipitate, keeping a perfect lattice coherency. Owing to this coherency, the newly formed crystal has the exactly same orientation as the old one. This process is repeated so that an extremely large number of nanocrystals with the same orientation are produced. There is abundant evidence supporting the above mentioned mechanisms [8]. These are listed as follows [8]. (1) The thickness of the bct precipitate formed below Tc is much larger than that for the case of the heating at Tc or slightly above Tc. (2) The spacing between the neighboring precipitates is $5-10 \mathrm{~nm}$ for the latter case, while $20-40 \mathrm{~nm}$ (a diameter of the nanocrystal) for the former case. (3) A bct precipitate is quite often observed at the amorphous-crystal interface as seen in Fig. 7 (b). (4) The enrichment of Ti atoms is detected at the bet precipitate by the energy dispersive analysis system (EDS). The $\mathrm{Ti}$ enrichment is also found in the amorphous facing the growth front of the crystallizing region. However, no such enrichment is detected in the amorphous region which is at the immediate neighbor of a newly formed bct precipitate at the amorphous-crystal interface.

\section{SHAPE MEMORY PROPERTIES OF THIN FILMS CONTAINING BCT PRECIPITATES}

\subsection{Ti-Ni binary alloys}

Owing to the coherency strains produced by bct precipitates, the shape memory properties are greatly improved for those thin films with the precipitates. Fig. 8 shows the strain vs. temperature curves under constant stresses for various heat treatment temperatures for Ti-48.2Ni. When a specimen is cooled from the temperature range of the parent phase under a constant load, the elongation starts to appear due to the martensitic transformation and is increased to a saturation level with decreasing temperature. On heating, the contraction occurs due to the reverse transformation and the elongation caused on cooling is completely recovered if any permanent strain, slip or deformation twinning, is not involved during the transformation cycle. The stress shown for each curve is the maximum stress over which the permanent strain is introduced so that the perfect shape recovery cannot be attained anymore. Curve (a) in this figure is for the specimens containing nanocrystals bounded by clustered bct plate precipitates, curve (b) for uniformly distributed bet plate precipitates, and curve (c) for orientated spherical $\mathrm{Ti}_{2} \mathrm{Ni}$ particles. It is quite clear that the resistance to the permanent strain is very high for those specimens containing bct precipitates. Especially, the specimen containing the unique nanocrystals shows very good shape memory properties such that the maximum recovery stress is three times greater than that for the specimen containing $\mathrm{Ti}_{2} \mathrm{Ni}$ precipitates and yet a large shape recovery strain of $5.6 \%$ is accompanied. Furthermore,

the hysteresis in the transformation temperature is quite small (about $30^{\circ}$ ) compared with the other cases.

Fig. 9 shows similar curves for Ti-47.3Ni, (a) being for the heat treatment at (Tc-50K) and (b) for the heat treatment for at Tc. As seen in these curves, the transformation hysteresis is very large compared with those in Fig. 8 although the maximum recovery stress is much higher. This is because the heat treatments near $\mathrm{Tc}$ in this alloy produce $\mathrm{Ti}_{2} \mathrm{Ni}$ precipitates besides the bct coherent precipitates. For Fig. 9 (a), the structures created by heating at this temperature consist of the nanocrystals bounded by clustered bct precipitates and randomly orientated $\mathrm{Ti}_{2} \mathrm{Ni}$ precipitates. The latter precipitates are considered to have formed directly from the amorphous before the crystallization into B2 structure takes place. For Fig. 9(b), three structures were produced by this heat treatment, namely, uniformly distributed bct plate precipitates, 
orientated $\mathrm{Ti}_{2} \mathrm{Ni}$ precipitates and randomly orientated $\mathrm{Ti}_{2} \mathrm{Ni}$ precipitates [10]. The orientated $\mathrm{Ti}_{2} \mathrm{Ni}$ precipitates are generated after crystallization into the $\mathrm{B} 2$ matrix and partially coherent with the matrix lattice [11]. Another interesting characteristic feature with Ti-47.3Ni in Fig. 9 is that the fracture occurs without accompanying any plastic deformation, so, if the external stress exceeds the maximum recovery stress, the sample is fractured instantaneously. In Fig. 9 we see two stages in the elongation curve on cooling; the first stage is due to the B2 to R-phase transformation and the second stage is caused by the Rphase to B19' transformation, starting from the point indicated by an arrow in the curve.

\subsection{Ti-Ni-Cu ternary alloys}

The microstructures produced in $\mathrm{Ti}-43.6 \mathrm{Ni}-6.2 \mathrm{Cu}$ and $\mathrm{Ti}-40.0 \mathrm{Ni}-5.7 \mathrm{Cu}$ by low temperature heat treatments are basically the same as those of $\mathrm{Ti}-48.2 \mathrm{Ni}$ except that the heat treatment slightly above $\mathrm{Tc}$ for Ti-40.0Ni-5.7Cu produces some amount of orientated spherical $\mathrm{Ti}_{2} \mathrm{Ni}$ particles besides the bct thin plate precipitates. The Tc temperatures for the former and latter alloys are $748 \mathrm{~K}$ and $754 \mathrm{~K}$, respectively. Figs. 10 (a) and (b) show the strain vs. temperature curves for various stresses; (a) for Ti-43.0Ni-6.2Cu, being heat treated at $698 \mathrm{~K}$ for $3.6 \mathrm{ks}$, and (b) for Ti- $40.0 \mathrm{Ni}-5.7 \mathrm{Cu}$, being heat treated at $723 \mathrm{~K}$ for $3.6 \mathrm{ks}$. These heat treatments produce for both the alloys the nanocrystals bounded by bet precipitates such as shown in Figs. 5-7. The strain vs. temperature curves in Fig. 10 were obtained in the following way. The stress was increased stepwise, starting from a very small stress such as 25-30 MPa. After each thermal cycle, the applied stress was increased by an amount of 30-50 MPa for the next run and the identical sample was
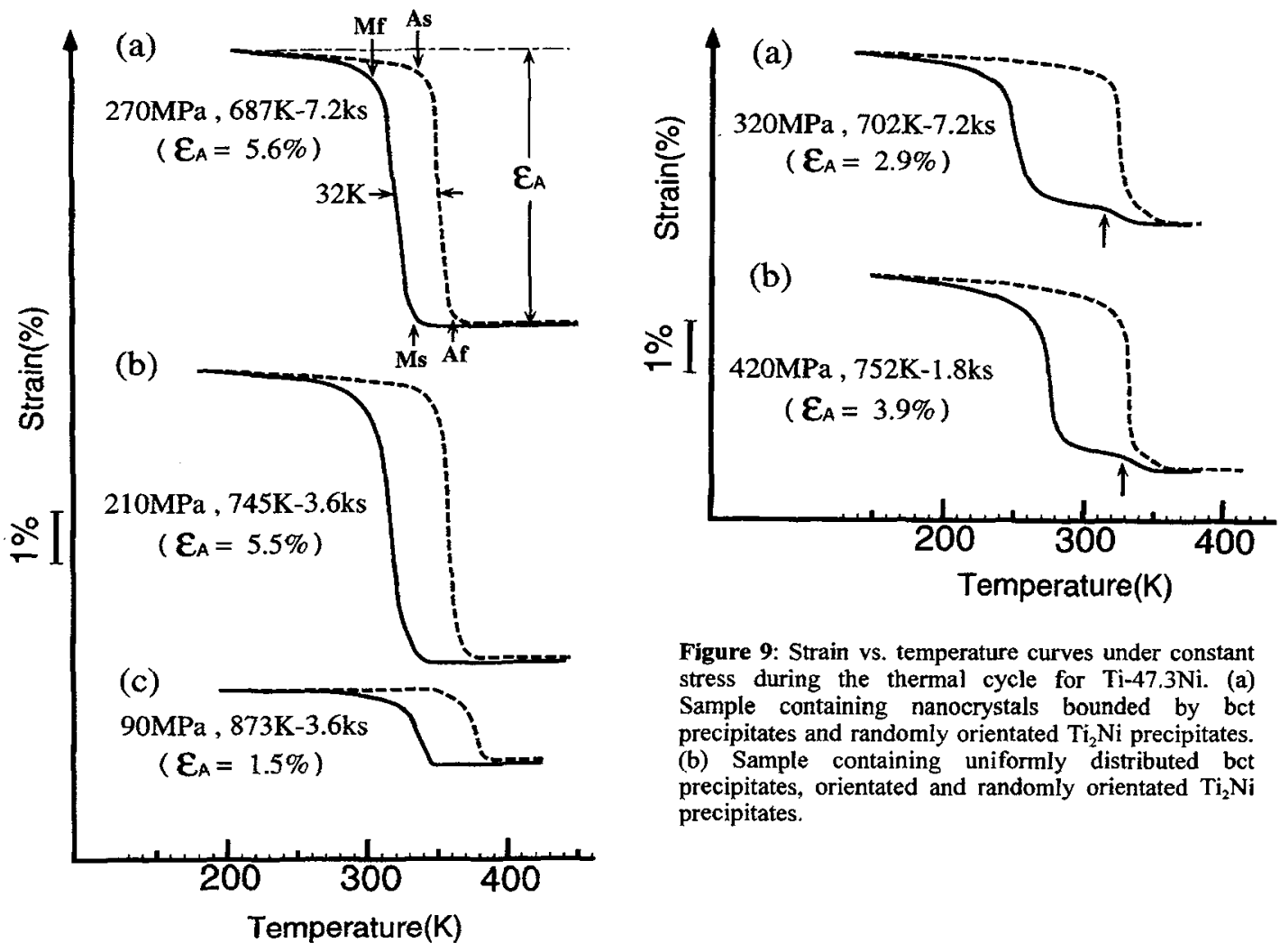

Figure 9: Strain vs. temperature curves under constant stress during the thermal cycle for Ti-47.3Ni. (a) Sample containing nanocrystals bounded by bct precipitates and randomly orientated $\mathrm{Ti}_{2} \mathrm{Ni}$ precipitates. (b) Sample containing uniformly distributed bct precipitates, orientated and randomly orientated $\mathrm{Ti}_{2} \mathrm{Ni}$ precipitates.

Figure 8: Strain vs. temperature curves under constant stress during the thermal cycle for Ti-48.2Ni. (a) Sample containing nanocrystals bounded by bet precipitates. (b) Sample containing uniformly distributed bct precipitates. (c) Sample containing orientated $\mathrm{Ti}_{2} \mathrm{Ni}$ precipitates. 
$\mathrm{Ti}-43.0 \mathrm{Ni}-6.2 \mathrm{Cu}, 698 \mathrm{~K}-3.6 \mathrm{ks}$

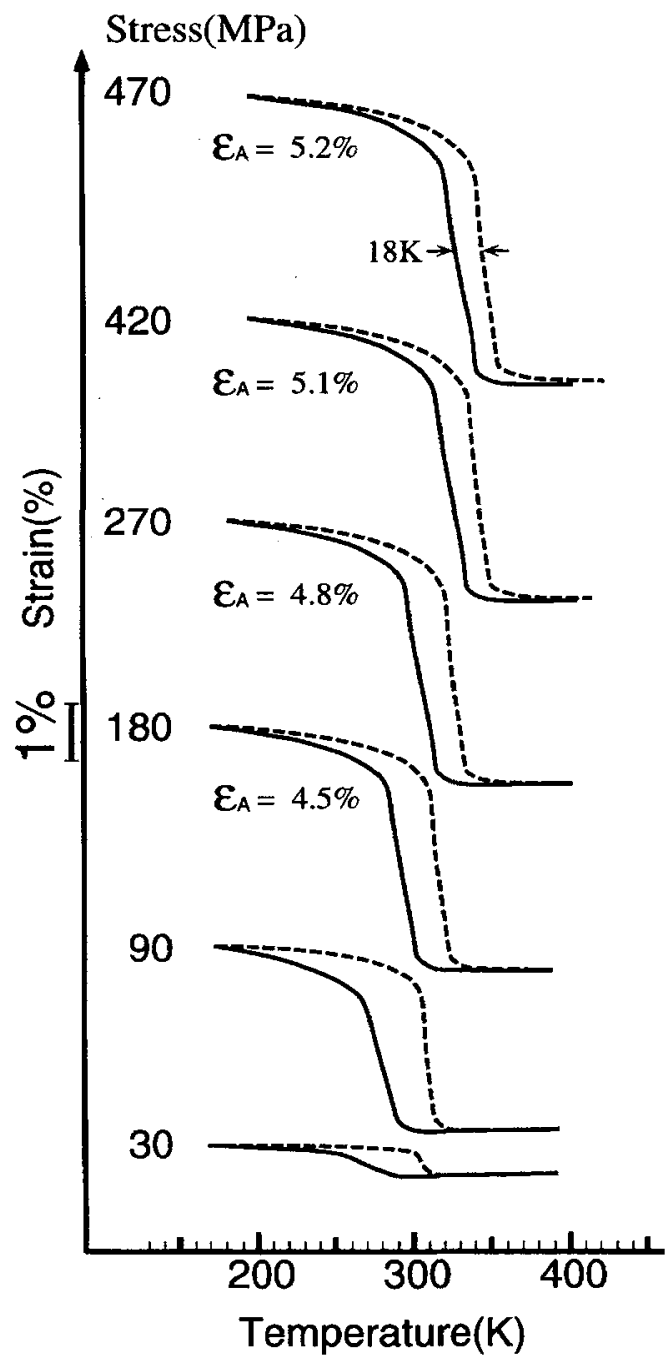

(a)
Ti-40.0Ni-5.7Cu , 723K-3.6ks

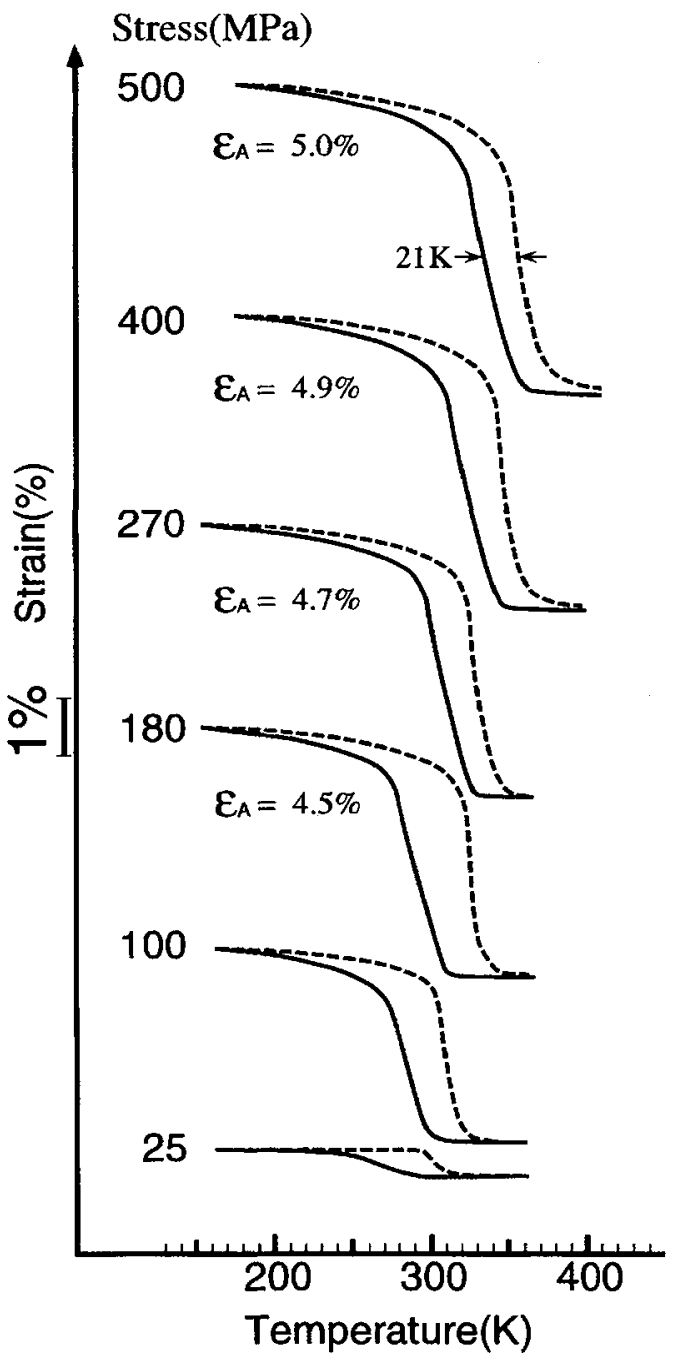

(b)

Figure 10: Strain vs. temperature curves under constant stress during the thermal cycle. Stress level is increased stepwise, using an identical specimen. (a) Ti-43.0Ni-6.2Cu heat treated at (Tc-50 K), (b) Ti-40.0Ni-5.7Cu heat treated at (Tc-31 K). Both the samples contain nanocrystals bounded by bet precipitates. 
employed throughout the whole transformation cycles. The curves only for the representative stresses are shown in Fig. 10. Comparing these curves with Fig. 8 (a), which is for Ti-48.2Ni having the same internal structures, i.e., the nanocrystals, we can point out the following differences between the binary and ternary alloys. (1) The maximum recovery stress is much higher for the ternary alloys, nearly two times greater than that for the binary alloy. (2) The transformation hysteresis is much smaller for the ternary alloys as is observed in the bulk specimen. As an origin for the increased recovery stress in the Ti-Ni-Cu alloys, we are obliged to attribute it to the addition of $\mathrm{Cu}$ because the internal structures are the same for both the alloys as mentioned above. Then what kind of effects of $\mathrm{Cu}$ addition to the Ti-Ni binary alloy would cause such an increase in recovery stress? It is hard to answer this question. The solution hardening due to $\mathrm{Cu}$ reported by Hashinaga et al. in the Ti-Ni-Cu alloy thin films [12] is ruled out as a possible cause because, firstly, there is no increase in the yield stress up to around $5 \% \mathrm{Cu}$ in their data and, secondly, the maximum recovery stress for the bulk Ti-45Ni-5Cu alloy is only $40 \mathrm{MPa}$ [13]. The origin for the remarkable strengthening effect by several percent $\mathrm{Cu}$ addition is not easy to understand, but it should be related with the bct coherent plate precipitates generated by low temperature heat treatments of Ti-rich $\mathrm{Ti}-\mathrm{Ni}-\mathrm{Cu}$ amorphous alloys, which is highly strained, partially disordered by replacement of $\mathrm{Ni}$ with $\mathrm{Cu}$ atoms and perfectly coherent with the matrix.

The most important feature seen in Fig. 10 is that the strain vs. temperature curves for Ti-43.0Ni6.2C and Ti-40.0Ni-5.7 Cu are almost the same for various levels of the applied stress; in other words, the shape memory properties are the same for these alloys with different $\mathrm{Ti}$ contents $(50.8 \% \mathrm{Ti}$ and $54.3 \% \mathrm{Ti})$. This is not true for the Ti-Ni binary alloy as seen in Figs. 8 and 9. For the case of uniformly distributed bct precipitates generated by heat treatments at $\mathrm{Tc}$ or slightly above Tc in the ternary alloys, the strain vs. temperature curves similar to those in Fig. 10 are also obtained although the maximum recovery stress is somewhat smaller.

\section{DISCUSSION}

As described in the previous section, excellent shape memory properties such as $500 \mathrm{MPa}$ shape recovery stress with about $5 \%$ recovery strain can be obtained for the Ti-Ni-Cu ternary alloys with the composition range of 51-54\% $\mathrm{Ti}$ if we utilize the bet precipitate formation. Besides these shape memory properties, it is readily seen from Fig. 10 that the transformation temperatures, Ms, Mf, As and Af, are approximately the same for the corresponding level of the applied stress in both the alloys of Ti-43.0Ni$6.2 \mathrm{Cu}$ and $\mathrm{Ti}-40.0 \mathrm{Ni}-5.7 \mathrm{Cu}$. In fact, the result of the plots of these transformation temperatures against the applied stress clearly revealed that the transformation temperatures are coincident with each other within $5 \mathrm{~K}$. This is quite a surprising feature when being compared with a very sensitive composition dependence of $\mathrm{Ms}$ in the ordinary Ti-Ni alloys. It is well known that, for Ti-Ni alloys, $1 \%$ change in the composition causes the Ms temperature change of about $100 \mathrm{~K}$. The reason for such compositioninsensitiveness of the transformation temperatures in these ternary alloys is considered to be due to two following factors. Firstly, the excess Ti content (over $50 \%$ ) will be absorbed in the bct precipitate, keeping the composition of the matrix nearly at the constant value of $50 \% \mathrm{Ti}$. Of course, it is possible to utilize the $\mathrm{Ti}_{2} \mathrm{Ni}$ precipitate formation for this purpose, but, in that case, only poor shape memory properties are obtained. Secondly, since the bonding force between $\mathrm{Ti}$ and $\mathrm{Ni}$ atoms in the ternary alloy may not be so strong as in the $\mathrm{Ti}-\mathrm{Ni}$ binary alloy, the tendency to form the $\mathrm{Ti}_{2} \mathrm{Ni}$ precipitate will be somewhat reduced so that a much greater number of the excess $\mathrm{Ti}$ atoms will be possible to remain in random solution in the matrix. On the contrary to the case of the ternary alloy, it seems that the $\mathrm{Ti}_{2} \mathrm{Ni}^{\mathrm{N}}$ precipitates are much easier to form in the binary alloy when the alloy composition is Ti-rich: as mentioned in the preceding section, low temperature heat treatments near Tc produce $\mathrm{Ti}_{2} \mathrm{Ni}$ precipitates besides the bct precipitates even for an excess Ti content of only $2.7 \%(\mathrm{Ti}-47.3 \mathrm{Ni})$.

In conclusion, we list the following conditions for developing composition-insensitive SMA thin films with high recovery stress and large recovery shape strain. (1) Ti-Ni based ternary alloys with Ti rich (more than $50 \%$ ) must be used. (2) The initial state before heat treatment must be amorphous. (3) The heat treatment must be done at low temperatures near the crystallization temperature, $\mathrm{Tc}$, preferably at 
(Tc-50 K).(4) The specimen must be directly heated from the amorphous state to this temperature. These conditions will be applicable also to $\mathrm{Ti}-\mathrm{Ni}-\mathrm{Pd}, \mathrm{Ti}-\mathrm{Ni}-\mathrm{Hf}$ and similar ternary alloys besides the present example of Ti-Ni-Cu alloys.

Finally the present author emphasize that the direct low temperature heat treatment from the initial amorphous state has two following additional benefits for practical application: firstly, it obviously reduces the production cost compared with the conventional two stage heat treatments of the Ni-rich Ti$\mathrm{Ni}$ based thin films, secondly and more importantly, the heat treatment near $750 \mathrm{~K}$ does not damage the base materials of silicon that is used for the sophisticated parts of micro-machines.

\section{Acknowledgments}

This paper is based on the work done in collaboration with Drs. T. Kikuchi, K. Ogawa of National Research Institute for Metals, Prof. S. Miyazaki of Tsukuba University and Messrs. T. Matsunaga and K. Yamazaki, Graduate Students of Tsukuba University. The author greatly appreciates their useful discussions and help to complete this review paper.

\section{References}

1. S. Miyazaki, A. Ishida and A. Takei, Proceedings of the International Conference on Martensitic Transformation, Montrerey, California, 1992, edited by C. M. Wayman and J. Perkins (Montrerey Institute of Advanced Studies, 1993), p. 983.

2. S. Miyazaki and A. Ishida, Proceedings of the International Conference on Martensitic Transformation, Bariloche, Argentina, 1998, edited by M. Ahlers, G. Kostorz and M. Sade, (Mat. Sci. \& Eng., 273-275, 1999) p. 106.

3. S. Kajiwara, T. Kikuchi, K. Ogawa, T. Matsunaga and S. Miyazaki, Phil. Mag. Lett. 74137 (1996).

4. S. Kajiwara, K. Ogawa, T. Kikuchi, T. Matsunaga and S. Miyazaki, Phil. Mag. Lett. 74, 395 (1996).

5. S. Kajiwara, T. Kikuchi, K. Ogawa, T. Matsunaga and S. Miyazaki, Proceedings of the 2nd International Conference on Shape Memory and Superelastic Technologies: Engineering and Biomedical Applications, Pacific Grove, California, 1997, edited by A. Pelton, D. Hodgson, S. Russell and T. Duerig, (SMST, California, 1997). p. 155

6. K. Ogawa, T. Kikuchi, S. Kajiwara, T. Matsunaga and S. Miyazaki, Proceedings of the 4th European Symposium on Martensitic Transformations, Enschede, Netherlands, 1997, edited by J. Beyer, A. Bottger, J. H. Mulder, ( J. PHYS. FRANCE 7 ,1997), C5-221.

7. T. Matsunaga, S. Kajiwara, K. Ogawa, T. Kikuchi and S. Miyazaki, Proceedings of the International Conference on Martensitic Transformation, Bariloche, Argentina, 1998, edited by M. Ahlers, G. Kostorz and M. Sade, (Mat. Sci. \& Eng,, A 273-275, 1999), p. 745.

8. T. Kikuchi, K. Ogawa, S. Kajiwara, T. Matsunaga, S. Miyazaki and Y. Tomota, Phil. Mag., A 78 (1998) 467.

9. T. Kikuchi, K. Ogawa, S. Kajiwara, T. Matsunaga, S. Miyazaki, Proceedings of the International Conference on Solid-Solid Phase Transformations, Kyoto, 1999, edited by M. Koiwa, K. Otsuka and T. Miyasaki, (The Japan Institute of Metals, 1999) p. 1092.

10. T. Matsunaga, S. Kajiwara, K. Ogawa, T. Kikuchi and S. Miyazaki, Proceedings of the International Symposium and Exhibition on Shape Memory Materials, Kanazawa, Japan, 1999, edited by T. Saburi, (Materials Science Forum Vols. 327-328, 2000), p. 175.

11. Ishida, A., Ogawa, K., Sato, M. and Miyazaki, S., Metall. Mater. Trans. A 28, 1985 (1997).

12. H. Hashinaga, S. Miyazaki, T. Ueki and H. Horikawa, Proceedings of the International Conference on Martensitic Transformation, Lausanne, Switzerland, 1995, edited by R. Gotthardt and J. Van Humbeeck (J. Phys. FRANCE IV, 1995) C8-689.

13. T. N. Nam, T. Saburi, K. Shimizu, Mater. Trans. Japan Inst. Met., 31, 959 (1990). 